\title{
Philosophiques
}

\section{Imaginaires sociaux et modernités multiples}

\section{Thomas McCarthy}

Volume 33, numéro 2, automne 2006

URI : https://id.erudit.org/iderudit/013895ar

DOI : https://doi.org/10.7202/013895ar

Aller au sommaire du numéro

Éditeur(s)

Société de philosophie du Québec

ISSN

0316-2923 (imprimé)

1492-1391 (numérique)

Découvrir la revue

Citer ce document

McCarthy, T. (2006). Imaginaires sociaux et modernités multiples.

Philosophiques, 33(2), 485-491. https://doi.org/10.7202/013895ar d'utilisation que vous pouvez consulter en ligne.

https://apropos.erudit.org/fr/usagers/politique-dutilisation/ 
Imaginaires sociaux et modernités multiples

\author{
THOMAS MCCARTHY \\ Northwerstern University \\ t-mccarthy@northwestern.edu
}

La récente étude de Charles Taylor sur les "imaginaires sociaux modernes » fait suite à une série d'essais antérieurs traitant de thèmes connexes ${ }^{1}$. Précédemment, lors d'une discussion sur l'unité cosmopolite et la diversité nationale, je me suis brièvement penché sur une des principales considérations de ces essais, à savoir la possibilité de modernités «alternatives » ou «multiples ", et j'ai soutenu que le champ des différentes modernités pourrait être plus restreint que ne le conçoit Taylor ${ }^{2}$. J'aimerais poursuivre ici cette discussion, dont la difficulté est que le livre recensé ici se concentre entièrement sur la modernité européenne. Mais lors d'un bref épilogue sur la "provincialisation de l'Europe ", Taylor suggère que son analyse des imaginaires sociaux modernes permet à leurs "particularités locales » d'émerger plus clairement et nous aide ainsi à "dépasser notre vision de la modernité comme un processus unitaire dont l'Europe est le paradigme ${ }^{3}$ ». C'est cette suggestion que je souhaite examiner.

Mes remarques porteront généralement sur le fait que son analyse se consacre trop exclusivement à l'herméneutique culturelle pour pouvoir nous assurer d'une telle chose, que le côté «matérialiste» des choses - qu'il admet à plusieurs reprises sans toutefois le discuter - devrait être reconnu à sa juste valeur avant que nous puissions risquer un tel jugement, et que lorsque cet aspect est mis de l'avant, la nature essentiellement politique de la tâche consistant à développer, soutenir et réconcilier de multiples modernités émerge plus clairement qu'elle ne le fait dans l'étude de Taylor.

1. L'analyse de la modernité européenne par Taylor se concentre, dans cet ouvrage, sur la famille d' 'imaginaires sociaux" qui en sont venus à imprégner les sociétés nord-atlantiques durant la période moderne et à

1. Modern Social Imaginaries, Durham, NC, Duke University Press, 2004. Dans les travaux antérieurs, voir tout spécialement: "Nationalism and Modernity", in R. McKim \& J. McMahon, dir., The Morality of Nationalism, Oxford, Oxford University Press, 1997, pp. 3155 ; "Conditions of an Unforced Consensus on Human Rights ", in J. Bauer \& D. Bell, dir., The East Asian Challenge for Human Rights, Cambridge, Cambridge University Press, 1999, pp. 124144 ; et "Two Theories of Modernity ", in D. Gaonkar, dir., Alternative Modernities, Durham, NC, Duke University Press, 2001, pp. 172-196.

2. "On Reconciling Cosmopolitan Unity and National Diversity ", in D. Goankar, dir., Alternative Modernities, pp. 197-235, esp. pp. 228-235.3.

3. Modern Social Imaginaries, pp. 195-196. Ci-après, les références à cet ouvrage seront insérées dans le texte, avec les numéros de pages, entre parenthèses. 
animer leurs pratiques caractéristiques et leurs institutions. Bien que l'on dise que ces imaginaires sociaux existent d'abord sous le mode d'un arrière-plan de compréhension partagée des situations et des pratiques sociales (24-5), ils sont typiquement informés par des conceptions de l'ordre moral de la société qui apparaissent d'abord sous la forme d'idées, lesquelles sont d'abord adoptées par quelques penseurs influents, infiltrent ensuite l'imaginaire social de strates plus larges et en viennent éventuellement à transmuer celui de la société entière (2, 24, 109). Les nouvelles théories de la loi naturelle de Grotius et de Locke en sont le principal exemple. Cette façon de représenter le changement historique semble inviter le genre de critiques que Marx a formulées contre l'idéalisme allemand. Taylor insiste donc dès le début sur le fait que son approche n'en est pas une qui oppose les «idées » aux " institutions » puisque les imaginaires sociaux sont en fait ce qui rend possible, en leur donnant un sens, les pratiques sociales et les institutions.

Je crois que ce genre d'objections est basé sur une fausse dichotomie, celle qui existe entre les idées et les facteurs matériels en tant qu'entités causales rivales. En fait, ce que l'on voit dans l'histoire humaine, ce sont des champs de pratiques humaines qui sont les deux à la fois, c'est-à-dire des pratiques matérielles [...] [et des] modes de raisonnement [...]. Ceuxci sont souvent inséparables [...] parce que les autocompréhensions sont la condition essentielle pour que la pratique ait le sens qu'elle a pour les participants (31-2).

Je trouve frappante la similarité avec l'argument "ontologique » soulevé par Gadamer aux dépends d'Habermas lors de leurs débats des années soixante : les compréhensions sont internes aux pratiques. Et la réponse d'Habermas semble également à sa place ici : "d'accord, toutefois il ne s'agit pas ici d'ontologie, mais de méthodologie, c'est-à-dire de savoir comment procéder pour analyser et expliquer le changement social. " Sur ce point, Taylor soutient qu'il n'y a pas qu'une seule sorte d'explication à donner — c'est-à-dire, pas de théorie générale du changement social — mais plutôt une myriade d'histoires causales complexes à raconter, dans lesquelles toutes sortes de facteurs jouent des rôles changeants $(33,41)$. Néanmoins, et cela est peut-être dû à la nature de cette étude particulière, le langage de Taylor donne souvent l'impression que les idées - sous la forme de conceptions de l'ordre moral — et les cultures — sous la forme des imaginaires sociaux - font la majeure partie du travail. Ainsi, certaines formes d'imaginaire social "sous-tendaient la montée de la modernité occidentale ", et une nouvelle conception de l'ordre moral de la société était centrale à cette modernité, conception dont la «mutation [...] dans notre imaginaire social est l'avènement de certaines formes sociales [...] qui caractérisent essentiellement la modernité occidentale » (2, italique ajouté). Par exemple, que l'on en vienne à voir la société comme une économie est un déplacement 
dans l'imaginaire social «forgé par » ou "dérivé de » cette nouvelle idée de l'ordre moral (76-7). De plus, la Révolution française et ses contrecoups faisaient intervenir de nouvelles formes et pratiques politiques « engendrées par» (spawned by) des théories de la souveraineté populaire (126).

Peut-être est-ce bien ce que Taylor avait en tête, mais je tends à penser qu'il s'agit d'un artéfact de l'approche herméneutique propre à la perspective culturelle qu'il adopte ici. Et ce qui m'encourage à en faire cette lecture, c'est non seulement les essais précédents, mentionnés cidessus, mais aussi la discussion de Taylor, dans cet ouvrage, sur les deux perspectives complémentaires à partir desquelles on voit la société moderne : comme un système de processus interconnectés, dotés de leurs propres lois - c.-à-d. comme une économie - et comme un peuple autogouverné, avec un espace de communication - par ex. une entité politique démocratique avec une sphère publique. La première est une perspective «objectifiante» de la vie sociale, la seconde nous imagine comme un agent collectif. "Les deux sont liées comme parties d'un même lot [...] La compréhension moderne de la société est irrémédiablement bifocale» (77). En conséquence, le politique est pensé comme «limité par l'extrapolitique, par différents domaines de la vie ayant leur propre intégrité et leur propre finalité ", de telle façon que l'action collective éclairée requiert normalement une solide analyse objective (164-7).

Dans cet ouvrage, toutefois, Taylor n'offre pas une telle vision bifocale; la perspective objectifiante de la société moderne est largement laissée pour compte.

Je n'y vois aucun problème en principe, pour autant qu'il soit clair qu'aucune conclusion concernant les changements sociaux ne peut être tirée de son étude herméneutique avant qu'une analyse objective complémentaire ne soit mise simultanément de l'avant. Ainsi, je résisterais à la suggestion que les "particularités locales ", sur lesquelles l'explication herméneutique attire notre attention, soient suffisantes pour écarter l'explication de "procédé unique » qui est avancée par les théoriciens sociaux classiques. Il ne s'agit pas que d'un autre désaccord théorique abstrait, puisque la question des modernités multiples est centrale pour le diagnostic de la conjecture mondiale actuelle et des chemins possibles allant au-delà de celle-ci. La lecture unidimensionnelle que fait Taylor de la modernité pourrait bien inciter ses lecteurs à adopter une perspective exagérément "culturaliste » sur ce qui est, selon moi, un problème politique impliquant des contraintes «matérialistes» significatives. 
2. L'approche que je privilégie sur la question des modernités multiples peut être introduite assez succinctement, en commentant les similarités et les différences entre Taylor et Dipesh Chakrabarty, dont le livre Provincializing Europe fournit son titre à l'épilogue de Modern Social Imaginaries ${ }^{4}$.

(i) Chakrabarty commence avec ce que l'on pourrait appeler le «fait» de la modernité culturelle mondiale et des contraintes pragmatiques que cette condition impose aux participants dans le discours des modernités multiples.

Le phénomène de la «modernité politique » — à savoir le règne des institutions modernes de l'État, de la bureaucratie et de l'entreprise capitaliste - est impossible à penser, où que ce soit dans le monde, sans invoquer certaines catégories et concepts dont les généalogies [...] portent toutes le poids de la pensée et de l'histoire européennes. Nul ne peut penser à la modernité politique sans ces concepts et d'autres concepts qui leurs sont reliés [...]. Cet héritage est maintenant mondial (PE, 2).

En conséquence de l'hégémonie européenne mondiale durant la période moderne, ces concepts et la vision universaliste de l'humanité qu'ils expriment sont «inévitables - et, en un sens, indispensables » (ibid.) Ils modèlent la pensée universitaire, maintenant mondiale elle aussi, dans l'histoire et les sciences humaines, y compris l'analyse et la critique de l'impérialisme occidental lui-même.

(ii) Toutefois, bien que la pensée européenne «soit maintenant l'héritage de tous ", elle est aussi "inadéquate pour nous aider à bien considérer les expériences de modernité politique dans les nations non occidentales » (PE, 16). Ainsi, la tâche consiste à provincialiser ou à décentrer l'Europe, non pas à rejeter la pensée européenne, "à laquelle nous devons notre propre existence intellectuelle ", mais à la repenser et à la renouveler "à partir de et pour ses marges ", qui sont plurielles et diverses (ibid.). En particulier, l'idéologie historiciste selon laquelle les modernités non occidentales ne font que reproduire le modèle occidental, quoique avec des retards plus ou moins grands de développement, et qui informe toujours la théorie et la pratique du développement dans la plupart des contextes nationaux et internationaux, doit être remplacée par des modes plus pluralistes de pensée des histoires passées et des possibilités présentes.

(iii) L'accord de Chakrabarty avec Taylor sur ce point va encore un peu plus loin. La lignée marxiste des "études subalternes", avec lesquelles il a été associé de près, le mène à concevoir la tâche qui nous

4. Provincializing Europe, Princeton, Princeton University Press, 2000. Ci-après « PE », avec le numéro de page entre parenthèses. 
incombe selon une double perspective: il cherche à combiner les approches analytique et herméneutique de l'enquête historique et sociale. Il est toutefois explicite quant au type de cadre analytique général qu'il veut déployer parallèlement aux interprétations et aux narrations locales :

Marx est critique pour l'entreprise, puisque sa catégorie «capital » nous donne une façon de penser à la fois à l'histoire et à la figure séculaire de l'humain à l'échelle mondiale, tout en faisant de l'histoire un outil critique pour comprendre le monde produit par le capitalisme ${ }^{5}$ (PE, 18).

(iv) La raison principale pour laquelle le marxisme, ou quelque autre forme de théorie sociale générale, est essentiel au discours des modernités multiples est ce que nous pourrions appeler le «fait» de la modernité sociale mondiale. Il n'y a pas que la pensée occidentale qui soit maintenant un héritage mondial. Le colonialisme européen et ses répercussions ont aussi propagé à travers le monde les pratiques et les institutions de la modernité économique, sociale et politique. Bien que celles-ci puissent ne pas être «inévitables » comme le sont certains éléments de la pensée européenne moderne, elles sont «irrésistibles » en un sens que Taylor lui-même a déjà expliqué comme suit:

D'un certain point de vue, la modernité est comme une vague qui inonde et engloutit une culture traditionnelle après l'autre. Si nous comprenons par modernité, entre autres [...] l'émergence d'une économie de marché industrielle, d'un État organisé bureaucratiquement, de modes de gouvernement populaire [...], les deux premiers changements, si ce n'est le troisième, sont, en un sens, irrésistibles. Celui qui néglige de les adopter, eux ou un bon équivalent fonctionnel, prendra tellement de retard dans les enjeux stratégiques qu'il sera conquis et forcé de procéder à ces changements de toute façon [...]. Il y a, dans les rapports de force, de bonnes raisons de favoriser la marche en avant de la modernité ainsi définie ${ }^{6}$.

C'était le cas avec la domination coloniale et impériale européenne durant la période moderne; et cela ne semble pas moins vrai des rapports de force néo-impériaux qui structurent les processus de mondialisation contemporains. Nous n'avons d'autre choix que de soupeser la possibilité de modernités multiples à partir de cette position délicate. C'est entre autres pour cette raison que Chakrabarty insiste pour que l'on joigne l'enquête herméneutique à l'analyse marxiste réformée, de façon à

5. Les références fréquentes de Chakrabarty à la « logique du capital » laissent croire qu’il ne partage pas la compréhension que Taylor a du matérialisme historique en tant que thèse sur la dominance de certains types de «motivations » - par ex., en ce qui concerne l'argent ou le pouvoir (p. 31). La compréhension de Chakrabarty semble plutôt se rapprocher de ce que Taylor caractérise ailleurs comme l'analyse des processus impersonnels montrant des systématicités quasinomiques (p. 76-77).

6. "Two Theories of Modernity», p. 182. 
arriver à un diagnostic proprement critique du présent et à une évaluation réaliste des possibilités qui restent ouvertes malgré les contraintes inhérentes aux conditions inéluctables de la modernité sociale et culturelle. Son but, nous dit-il, est de "trouver une forme de pensée sociale qui adopte la raison analytique dans la poursuite de la justice sociale, mais qui ne lui permet pas d'effacer la question de l'hétérotemporalité de l'histoire du sujet moderne » (PE, 239). Comme Taylor, je suis sceptique quant à la possibilité de résoudre sur le plan de la pensée sociale les tensions entre universalité et particularité, et je regarde plutôt du côté des pratiques de dialogue interculturel, y compris les pratiques politiques, pour poursuivre la dialectique de la convergence et de la divergence. Mais le discours de la modernité globale doit prendre en considération les racines systémiques des inégalités massives qui sont encore caractéristiques d'un tel dialogue, si l'agentivité collective doit être informée par l'analyse objective.

(v) Le discours général sur les causes et les remèdes de l'injustice globale appartient irrémédiablement au domaine du politique. Chakrabarty nous rappelle que la tension entre le «pas encore » du colonialisme et le «maintenant» du nationalisme anticolonial a été pour ainsi dire supplantée, non pas dans la théorie développementale, où elle subsiste, mais dans la pratique politique (PE, 8-10). Pour les mouvements de masse, le besoin de politiser la paysannerie et pour les démocraties de masse celui de leur octroyer une pleine citoyenneté a engendré la modernité politique des classes subalternes et le remplacement politique des théories développementales les concernant. De même, il me semble, les luttes politiques pour la justice mondiale doivent procéder en présupposant, de façon pratique, un statut égal et une pleine participation de tous les peuples.

3. Laissez-moi conclure ces brèves remarques en tirant de ce qui précède quelques conclusions provisoires sur le discours des modernités multiples.

(i) Le fait ou la condition de la modernité culturelle mondiale signifie que les points de départ herméneutiques de l'interprétation des cultures particulières sont toujours d'abord informés par les concepts, principes et idéaux de base de la culture occidentale. Plusieurs de ces derniers - par. ex., ceux qui sous-tendent les études historiques et les sciences humaines - figurent en tant que présuppositions pragmatiques inévitables dans, et donc en tant que contraintes qui rendent possible le discours des modernités multiples lui-même ${ }^{7}$.

7. Mais, comme le note Taylor, d'autres « facettes de la constellation moderne » sont des particularités de la culture moderne qui se sont faites passer pour des "caractéristiques vraiment universelles de la modernité » ( "Two Theories of Modernity », pp.179-81). Les distinguer les unes des autres constitue toutefois la tâche des modes de discours critiques-réflexifs qui sont eux-mêmes typiquement modernes. 
(ii) Le fait, ou la condition, de la modernité culturelle mondiale signifie que, pour survivre et prospérer, les sociétés doivent réussir à s'adapter aux environnements technologiques, économiques et politiques modernisés dans lesquels elles se retrouvent, qu'elles le veuillent ou non. Cela pose d'inévitables contraintes sur l'autocompréhension et l'auto-organisation de n'importe quel agent collectif qui espère éviter d'être perpétuellement dominé par les autres.

(iii) La provincialisation ou le décentrement de l'Europe n'est pas d'abord une tâche herméneutique, mais une tâche politique, qui s'élabore autour de la réduction des énormes disparités de richesse et de pouvoir qui structurent encore les relations entre les anciennes sociétés coloniales et les sociétés post-coloniales. Les modes d'agentivité collective propres à cette tâche nécessitent un input continu, non seulement d'autocompréhension herméneutique, mais aussi d'analyse objective et de critique informée.

(iv) Au terme de l'analyse, la portée de la divergence culturelle au sein de la convergence globale sera fortement dépendante de la mesure selon laquelle les dynamiques mondiales d'accumulation de capital pourront être politiquement maitrisées et gérées. Les formes de gouvernance globales que cela nécessite devraient favoriser la diversité multiculturelle et multinationale, et faire de l'espace à un développement culturel et national autonome.

(v) La délicate situation de la modernité politique appelle à la construction d'un ordre mondial cosmopolite, qui nécessite à son tour une convergence mondiale suffisante et un chevauchement suffisant entre les cultures légales et politiques pour légitimer les structures de gouvernance transnationales. Pour cela, les formes démocratiques de participation, dont le potentiel d'universalité semble mis en doute par Taylor, pourraient bien être nécessaires. Mais je ne mesure pas jusqu'où, finalement, nous serions en désaccord sur ce point — ni, du reste, sur les autres points que j'ai soulevés. 